\title{
THE NEURON AS A LIE GROUP GERM AND A LIE PRODUCT*
}

\author{
BY \\ WILLIAM C. HOFFMAN \\ Oregon State University
}

\begin{abstract}
A basic Lie group derived earlier that describes the "constancies" of visual perception is extended to one more dimension to take into account the nonlinear flow of an afferent volley of nerve impulses through the layers of the visual cortex. One is then led, via the usual determination of the solutions of a Lagrange partial differential equation in terms of an associated Pfaffian system of ordinary differential equations, to a correspondence between neuron cell body, Lie group germ, and critical point of the system of ordinary differential equations governing the orbits. The local phase portraits of the latter bear a marked resemblance to one or the other of the neuron types defined by Sholl. Since "brains are as different as faces", the concept of structural stability plays an important role in analyzing the connectivity of the neural network. Finally, Lukasiewicz's theory of parentheses is used to obtain a graph-theoretic representation of the Jacobi identity, which then serves to explain the branching of neuronal processes (dendrites).
\end{abstract}

1. Introduction. It is a commonplace how von Neumann's interest in digital computer design is supposed to have evolved out of Pitts and McCulloch's work on the perception of auditory and visual forms. Pitts and McCulloch were perhaps the first to recognize in this paper [28] that the various invariances of perception require, for their proper mathematical expression, the action of transformation groups upon the visual field of view. Of course many eminent scientists, ranging from Poincaré and Helmholtz to Akishige [1], [2], Cassirer [7], Culbertson [12], [13], Rashevsky [34], Rosenblatt [36], and Wiener [40], also recognized that groups were involved in perception. For example, the analysis of the psychological variables themselves (the parameter groups) in terms of finite transformation groups by Akishige and his school is definitive. However, in none of these studies was the combined group-manifold structure essential to understanding the perceptual invariances upon the visual field of view itself, namely, infinitesimal transformations in terms of Lie groups, introduced. The result was that Pitts and McCulloch, for instance, outstripped by several orders of magnitude the total number of neurons available in the entire cortex in setting up a functional model for invariance of visual perception under translation and dilatation of the visual image.

In a way, the success of the digital computers that grew out of Pitts and McCulloch's pioneering work has led us down the garden path, for the brain appears much more like a hybrid than a digital computer. The all-or-none ("spike") response of the axons is relatively less important than the graded response (Bishop [5]; Bullock [6]). The graded response is essentially analogue rather than digital in nature, and appears to be much more involved in higher mental faculties than the spike discharge.

Pitts and McCulloch also recognized that what we see are "geometric objects" in the strict mathematical sense (Kucharzewski and Kuczma [20]), and this immediately argues that a differential-geometric, "smooth" sort of perception must be involved.

*Received November 9, 1966. 
As Pribram [30] has put it in another context, "The brain, as more and more experimenters find, is truly made to accomplish what it must, behavior being what it is." To this we might add that the visual posterior intrinsic system (Pribram [29]) has developed so that we "see what we have to see, the geometric nature of our visual world being what it is. This feature, taken together with the characteristic features of the visual Gestalt, experiments on the recognition of defocussed images, and the cortical cytoarchitecture, strongly suggests that the mental "computer" operates more according to statistical decision procedures for Lie transformation groups and coset fibre bundles than any logic, recursive function theory notwithstanding. As we shall see, the cortex processes-or better, "preprocesses"-visual stimuli in such a way as to impose the various invariances of perception via differential flows that are consistent with known histology and cytoarchitecture. The cortical neurons do this by "dragging the flow" of visual contours along the orbits of Lie transformation groups, and it is to a discussion of the nature and role of Lie transformation groups that we now turn our attention.

2. Lie transformation groups. A Lie group is at the same time a manifold (which can be thought of in the present context as a surface imbedded in 3-space) and a group. The group structure must be consistent with the differentiable structure (Munkres, [25]). Two properties of the visual manifold will be of prime importance to us. The first is that it is compact, and so possesses a finite covering of open sets (neighborhoods). This property relates very directly to the finiteness of neuron structure and the finite limits of visual acuity. The second is that any open set in this finite covering is diffeomorphic to some open set in Euclidean space of the same dimensionality. Thus we can bypass the very difficult problem of the metric tensor on the subjective, variable visual manifold and consider instead its projection on "flat" Euclidean space, so far as the action of the Lie groups is concerned.

According to Lie's First Fundamental Theorem, a continuous transformation group is completely defined by the corresponding set of lie derivatives, the latter being of the form

$$
\mathfrak{L}_{\rho}=\sum_{i=1}^{n} f_{i \rho}\left(x_{1}, \cdots, x_{n}\right) \frac{\partial}{\partial x_{i}} \quad(\rho=1,2, \cdots, r) .
$$

In the present context, the lie derivative is simply a directional derivative, which, as we have noted, drags the "flow" along the orbits, or streamlines (Guggenheimer, [17]). Thus the action of a finite transformation group over a manifold is equivalent to the action of the infinitesimal transformations along the group orbits that fill out the manifold. That is, the Lie derivative generates the global transformation group over the full manifold by means of infinitesimal transformations within neighborhoods, the latter providing a finite covering of the surface. This local vs. global correspondence enables us to escape the difficulty encountered by Pitts and McCulloch with respect to the number of neurons available and, in the process, set up a correspondence between the local (microscopic, or histological) and global (psychological) scales of perception.

3. The basic Lie algebra $\mathfrak{B}$ of visual perception. The way to proceed further is indicated by the various "constancies" of visual perception and by form memory, which is really nothing but another name for the invariance of perception of particular forms under time translations. These perceptual invariances are collected in Figure 1. The ones in parentheses are not to be found as such in the psychological literature, 


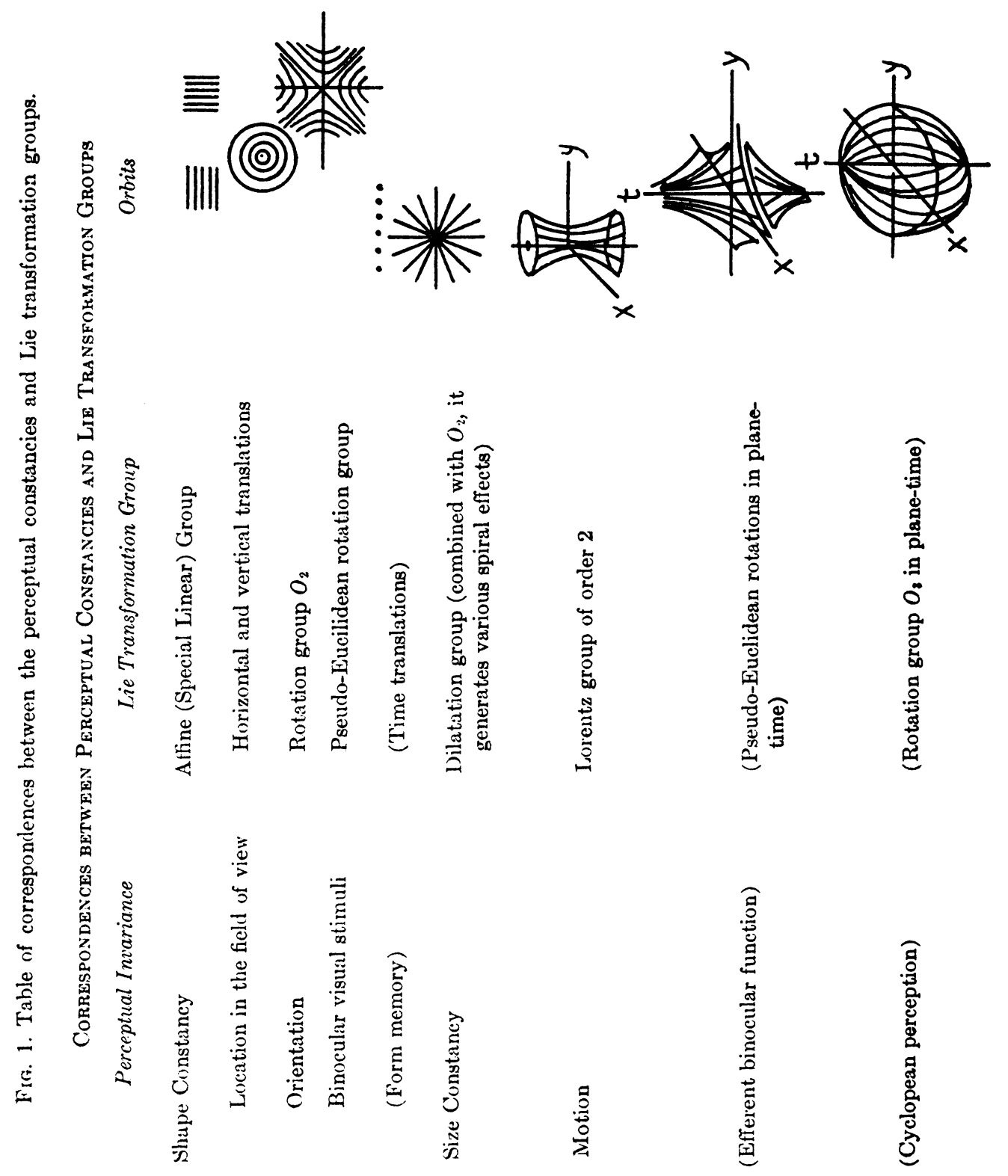


but must be present to avoid violating Lie's Second Fundamental Theorem. Also shown in the Figure are the corresponding Lie groups. Figure 2 gives the multiplication table for the Lie algebra involved, and the Lie derivatives that generate the group are shown in the cutout section. The latter are formed from the basic building blocks of the General Linear Group $\mathrm{GL}(3, R)$ in the same order as the developmental sequence of vision in human infant and kitten. All this has appeared in a recent paper [18], and the interested reader is referred to this publication for more details.

Most neurophysiologists will accept the possibility that the brain may be able to compute sums and differences and perhaps even products, but will balk strongly at any higher order functions. They will question such linear combinations of partial derivatives as those of Figure 2. Nonetheless such operations come about very simply when viewed as generating the orbits of Lie groups, and in a way consistent with known neurohistology in octopus [41], rat, cat, and monkey [10], and human infant [33]. The

\begin{tabular}{|c|c|c|c|c|c|c|c|c|c|c|c|c|c|c|c|}
\hline$\left[\alpha_{i}, \alpha_{j}\right]$ & $\sigma_{x}$ & $\alpha_{y}$ & $\alpha_{t}$ & $\mathcal{L}_{B}$ & $\mathcal{L}_{B 1}$ & $L_{B 2}$ & $L_{m}$ & $\alpha_{m 1}$ & $\alpha_{m 2}$ & $L_{M}$ & $\mathcal{L}_{M 1}$ & $\alpha_{M 2}$ & $L_{b}$ & $L_{s}$ & $\mathcal{L}_{r}$ \\
\hline$L_{x}$ & 0 & 0 & 0 & $\mathcal{L}_{x}$ & $-\mathcal{L}_{x}$ & 0 & $-\mathcal{L}_{y}$ & $L_{t} / c$ & 0 & $-L_{y}$ & $C_{t} / c$ & 0 & $L_{y}$ & $\mathcal{L}_{x}$ & $L_{y}$ \\
\hline$\delta_{y}$ & 0 & 0 & 0 & $-L_{y}$ & 0 & $-\alpha_{y}$ & $L_{x}$ & 0 & $L_{t} / c$ & $\mathcal{L}_{x}$ & 0 & $L_{t} / c$ & $L_{x}$ & $L_{y}$ & $-L_{x}$ \\
\hline $\mathcal{L}_{t}$ & 0 & 0 & 0 & 0 & $\mathcal{L}_{t}$ & $\mathcal{L}_{t}$ & 0 & $c \mathcal{L}_{x}$ & $c 5$ & 0 & $-c L_{x}$ & $-c L_{y}$ & 0 & 0 & 0 \\
\hline$\Sigma_{B}$ & & & 0 & 0 & 0 & 0 & $-2 L_{b}$ & $\delta_{M 1}$ & $C_{M 2}$ & $-2 C_{b}$ & $\mathcal{L}_{m 1}$ & $-L_{m_{2}}$ & $2 L_{r}$ & 0 & $2 C_{6}$ \\
\hline$C_{B 1}$ & & & & 0 & 0 & 0 & $\alpha_{b}$ & GNII & 42 & $L_{b}$ & $-2 \varepsilon_{m 1}$ & $-S_{m 2}$ & $L_{M}$ & 0 & $-\alpha_{6}$ \\
\hline$\alpha_{B 2}$ & & & & & 0 & 0 & $2 b$ & $-L_{M 1}$ & $-2 C_{M 2}$ & $-C_{b}$ & $-\alpha_{m}$ & $-2 \alpha_{\text {me }}$ & $L_{r}$ & 0 & $\alpha_{b}$ \\
\hline $\mathcal{L}_{m}$ & & & & & & $\alpha_{b}$ & 0 & $L_{m 2}$ & $-\alpha_{m 1}$ & 0 & $\alpha_{M 2}$ & $-\alpha_{M_{1}}$ & $-2 C_{B}$ & 0 & 0 \\
\hline$r$ & & & & & & & & 0 & $-L_{m}$ & $-L_{m 2}$ & $2 c_{B 1}$ & $-\alpha_{b}$ & $-L_{M 2}$ & $-L_{M I}$ & $L_{m 2}$ \\
\hline $\mathcal{L}_{m 2}$ & & & & & & & & $L_{m}$ & 0 & $\mathcal{L}_{m 1}$ & $-\alpha_{b}$ & $2 C_{B 2}$ & $-L_{M 11}$ & $-L_{M 2}$ & $-L_{\text {ntr }}$ \\
\hline $\mathcal{L}_{M}$ & & & & & & & & & $\alpha_{m l}$ & 0 & $\alpha_{M 2}$ & $-\alpha_{M_{1}}$ & $-2 \mathcal{L}_{B}$ & 0 & 0 \\
\hline$\alpha_{M 1}$ & & & & & & & & & & $-S_{M 2}$ & 0 & $L_{M}$ & $-\alpha_{m 2}$ & $-L_{m 1}$ & $L_{M 2}$ \\
\hline$\alpha_{M 2}$ & & & & & & & & & & $-M$ & $-\alpha_{M}$ & 0 & $-L_{m 1}$ & $x_{m 2}$ & $-x_{M 1}$ \\
\hline$\alpha_{b}$ & & & & & & & & & & & & $\mathcal{L}_{m+1}$ & 0 & 0 & $-2 C_{B}$ \\
\hline$\alpha_{5}$ & & & & & & & & & & & & & 0 & 0 & 0 \\
\hline & & & & & & & & & & & & & $2 L_{B}$ & 0 & 0 \\
\hline
\end{tabular}

Fra. 2. Multiplication table for the basic Lie algebra $\mathfrak{B}$ of mature visual perception. The parameter $c$ is the limiting value of the velocity of propagation of a nerve impulse through the cortex. 
orbits of $\mathfrak{L}_{x}, \mathfrak{L}_{y}$, and $\mathfrak{L}_{t}$ are, respectively $\equiv$ ||III, and some kind of ticks of a neurophysiological clock (presumably the spontaneously discharging neurons (Jung's type A)). The orbits of the operators $x(\partial / \partial x), y(\partial / \partial y), t(\partial / \partial t)$ are the same. However, $y(\partial / \partial x)$ and $x(\partial / \partial y)$ exhibit a double-valuedness, i.e., two parallel straight lines constitute the orbits in these cases. For example the integral curve associated with $(d x) / y=(d y) / 0$ is $y= \pm c$. Graphically, as they would appear in Golgi-Cox preparations of tangential sections of cortex, such parallel straight lines would be indistinguishable from the linear orbits of the simple translation operator $\mathfrak{L}_{x}$. Now the Lie product operation more or less automatically generates the Lie algebra of mature binocular perception [18] from the basic building blocks of $\mathrm{GL}(3, R)$ and the Lorentz group governing motion invariances upon the visual manifold. Thus the complicated looking Lie derivatives that appear in the cutout on Fig. 2 are generated quite simply via graphical integration over the local orbits of the Lie groups involved. This principle is not only consistent with the histological studies of the plexiform layer of the cortex in octopus, rat, cat, monkey, and human that were cited above, but also with the result of stimulation of the surface of the brain in open-skull operations [28], patterns evoked by flicker stimulation [38] and LSD, the nature of MacKay's complementary after images, and the aesthetic satisfaction imparted by Optical Art [19]. In assessing such correspondences, it is necessary not to lose sight of the fact that the Lie derivative is a local, infinitesimal operator that acts over a small neighborhood, in the process generating a whole manifold.

4. The orbits of Lie group germs as local phase portraits. The Lie derivatives shown in Fig. 2 operate in the plane, not space. However, the "flow" of an afferent volley through the layers of the cortex has an intrinsic three-dimensional character so far as its spatial behavior is concerned. An afferent volley coming from the secondary optic radiation terminates largely in layer IV of the cortex, undergoes some kind of "circulating neuronal activity", and thence flows up to the cortical surface, back down again and then becomes efferent or corticocortical. In the mature higher animal this cortical "flow" of nerve impulses is associated with a triphasic electrocortical response [4], [22], [24]: surface positive-negative-positive afterdischarge, coupled with the occurrence of a deep negative potential situated primarily in layer IV. The essential point for our purposes is that the electrocortical response to an afferent volley is nonlinear and propagates in three-dimensional fashion through the depths of the cortex.

These two features-the nonlinearity and the three-dimensional character of the flow-argue that the orbits will be determined, in the depths of the cortex, by a differential system of the generic form

$$
\frac{d}{d \tau}\left(\begin{array}{l}
x \\
y \\
z
\end{array}\right)=\left(\begin{array}{c}
f_{1}(x, y) \\
f_{2}(x, y) \\
\zeta(x, y, z)
\end{array}\right)=\left(\begin{array}{lll}
a_{1} & b_{1} & 0 \\
a_{2} & b_{2} & 0 \\
a_{3} & b_{3} & 0
\end{array}\right)\left(\begin{array}{l}
x \\
y \\
z
\end{array}\right)+\left(\begin{array}{c}
g_{1}(x, y) \\
g_{2}(x, y) \\
g_{3}(x, y, z)
\end{array}\right),
$$

where $g_{3}(x, y, z)=z^{2 n} g(x, y)$, reflecting the nonlinear character of the cortical response to an afferent volley, as described above, and the upward propagation (positive $z$ ) from the local origin in layer IV. Of course, the "flow" reverses and becomes downward and/or intracortical once it reaches the plexiform layer, and so properly we should be dealing with a dynamical system describing the "flow" in a toroid with handlebodies. The present analysis should therefore be regarded in the light of a "first cut." 
The differential system (2) can be written in the vector form

$$
d \mathrm{x}_{3} / d \tau=A_{3} \mathrm{x}_{3}+\mathrm{g}_{3},
$$

where the column vector $\mathbf{x}_{3}=(x, y, z)^{\imath r}, A_{3}$ is the $3 \times 3$ matrix on the right in (2), and $g_{3}$ is the column vector of the $g$ 's in the same equation. It is well known that the behavior of the phase portrait of such a system as (3) in the neighborhood of a critical point is determined by the linear approximation

$$
d \mathrm{x}_{3} / d \tau=A_{3} \mathrm{x}_{3},
$$

which in turn can be reduced to canonical form by introducing the Jordan canonical form of the matrix $A_{3}$ :

where

$$
d \mathfrak{u}_{3} / d \tau=J_{3} \mathfrak{u}_{3},
$$

$$
J_{3}=T A_{3} T^{-1}, \quad \mathbf{x}_{3}=T^{-1} \mathbf{u}_{3}
$$

for some real nonsingular matrix $T$.

Such a program has been carried through by Mendelson [23] in determining the local phase portrait of such a system as (3) when the matrix $A_{3}$ is "well behaved" (all the eigenvalues have the same sign). The basic building blocks for the local phase portrait in such a case turn out to be what Mendelson calls "fan cones" and "saddle cones", which combine in ways that bear a striking resemblance to pyramidal and stellate neurons. However, Mendelson's analysis can be regarded as applying only to two of the cases of (3) that follow from the Lie derivatives defining the basic group of visual perception given in Fig. 2.

5. Comparison of local phase portraits and neuron morphology. We therefore pass to a case by case analysis of the Lie derivatives occurring in Fig. 2 that have solely a spatial character. Our object will be to compare the local phase portrait of the corresponding system (3) in the neighborhood of a critical point with neuron morphology in the vicinity of the perikaryon. Only those Lie derivatives with a purely spatial character will be considered because only these can be compared with the static, frozen picture of neuron structure and function provided by a Golgi-Cox preparation [37]. In this comparison we shall make use of Sholl's classification of cortical neurons into seven types, four pyramidal and three stellate, on the basis of "connectivity" (morphology) and Colonnier's study of the three dimensional morphology of cortical neurons.

The morphological classification of the types of cortical neurons determined by Sholl [37] is shown in Fig. 3. These "canonical neurons" are two dimensional as shown, but we know from the histological studies of Colonnier [10] and Gihr [16] that the pyramidal cells possess a roughly cylindrical symmetry and the stellate cells exhibit a radial symmetry, either disk-shaped or (prolate) spheroidal. Sholl's classification includes all but about $1 \frac{1}{2} \%$ of the cortical neurons, the "inverted pyramids" being the exception. As we shall see, even these emerge naturally from our analysis.

Let us consider first of all the local phase portrait corresponding via (3) (or (2)) to $\mathfrak{L}_{B}=x(\partial / \partial x)-y(\partial / \partial y)$. Then $A_{3}$ and $J_{3}$ take the forms

$$
A_{3}=\left(\begin{array}{ccc}
1 & 0 & 0 \\
0 & -1 & 0 \\
a_{3} & b_{3} & 0
\end{array}\right) \quad \text { and } \quad J_{3}=\left(\begin{array}{ccc}
1 & 0 & 0 \\
0 & -1 & 0 \\
0 & 0 & 0
\end{array}\right) \text {. }
$$




\begin{tabular}{|l|l|l|}
\hline & Typo & \multicolumn{1}{|c|}{ Description } \\
\hline & $P_{1}$ & $\begin{array}{l}\text { Pyramidal ooll with unbreacbed ason } \\
\text { to whito mattor }\end{array}$ \\
\hline
\end{tabular}

Fra. 3. Diagrams and descriptions of the principal types of neuron found in the cerebral cortex (after Sholl [37]).

The solution of the system (5) then yields the vector

$$
\mathbf{u}_{3}=\left(c_{1} e^{r}, c_{2} e^{-r}, c_{3}\right)^{\imath r},
$$

from which it follows that $u v=$ const., i.e. that the projection of the local trajectories on the $u, v$-plane are rectangular hyperbolas. Determining now the solution vector $\mathbf{x}_{3}$ according to the second of formulas (6), one finds

$$
\mathrm{x}_{3}=\left(u,-v, a_{3} x-b_{3} y+c_{3}\right)^{\imath r} .
$$

Further, because of the vanishing of one eigenvalue of the matrix $A_{3}$, we see that we shall have a "line of singular points" [35]. Because this is a generic feature of the matrix $A_{3}$, this is a feature that we shall encounter in all our phase portraits. It is exactly consistent with the layering and columnar structure of the cortex, and here perhaps we have another feature of the theoretical model that is strongly relevant to cytoarchitecture.

The orbits of $\mathfrak{L}_{B}$ thus all lie on a linear array of hyperbolic sheets (integral manifolds) like that of Fig. 4.

The paths in the upper sheet of the hyperboloid in Fig. 4 strongly resemble the appearance of a pyramidal neuron near the perikaryon itself. Well away from the perikaryon dendritic ramification complicates the morphological picture considerably, and we shall have more to say in this connection later when we discuss the Lie product aspect of neuron morphology. The lower sheet of the hyperboloid presumably provides an explanation of the morphology of the only kind of neurons that were omitted by Sholl in his classification, the so-called "inverted pyramids".

As noted above, however, the local phase portrait consists of a line of singular points, and thus the actual cortical counterpart of the processing by $\aleph_{B}$ would be a string of neurons, staggered, arranged in columns, or in layers. Only the latter two configura- 
tions appear to have been studied in the usual histological examination of sagittal and tangential sections of cortex.

A similar approach to the local phase portrait of the Lie derivative corresponding to afferent binocular function $\mathfrak{L}_{b}=y(\partial / \partial x)+x(\partial / \partial y)$ may be based upon the matrices

$$
A_{3}=\left(\begin{array}{lll}
0 & 1 & 0 \\
1 & 0 & 0 \\
a_{3} & b_{3} & 0
\end{array}\right) \quad \text { and } \quad J_{3}=\left(\begin{array}{ccc}
1 & 0 & 0 \\
0 & -1 & 0 \\
0 & 0 & 0
\end{array}\right)
$$

The rest of the analysis, employing Eqs. (4)-(6), leads to the solution vectors

$$
(u, v, w)=\left(c_{1} e^{\tau}, c_{2} e^{-\tau}, c_{3}\right),
$$

and, when transformed back into the $x, y, z$-space,

$$
(x, y, z)=\left(\frac{1}{2}(u+c / u), \frac{1}{2}(u-c / u), b_{3} x+a_{3} y+c_{3}\right) .
$$

Thus $u v=$ const. $=x^{2}-y^{2}$, and although the same type of hyperbolic family in the $u, v, w$-space is encountered as that of $\mathfrak{L}_{B}$, the configuration in the original space is like that shown in Fig. 5. The morphology of the local phase portrait in this case resembles that of either pyramidal or stellate neurons, depending upon which portion of the branches of the hyperbolas correspond to the neighborhood of a perikaryon, the inclination of the plane in which they lie, and how many members of the family of hyperbolas are involved.

For the Lie derivative associated with the phenomenon of size constancy

$$
\mathfrak{L}_{s}=x(\partial / \partial x)+y(\partial / \partial y)
$$

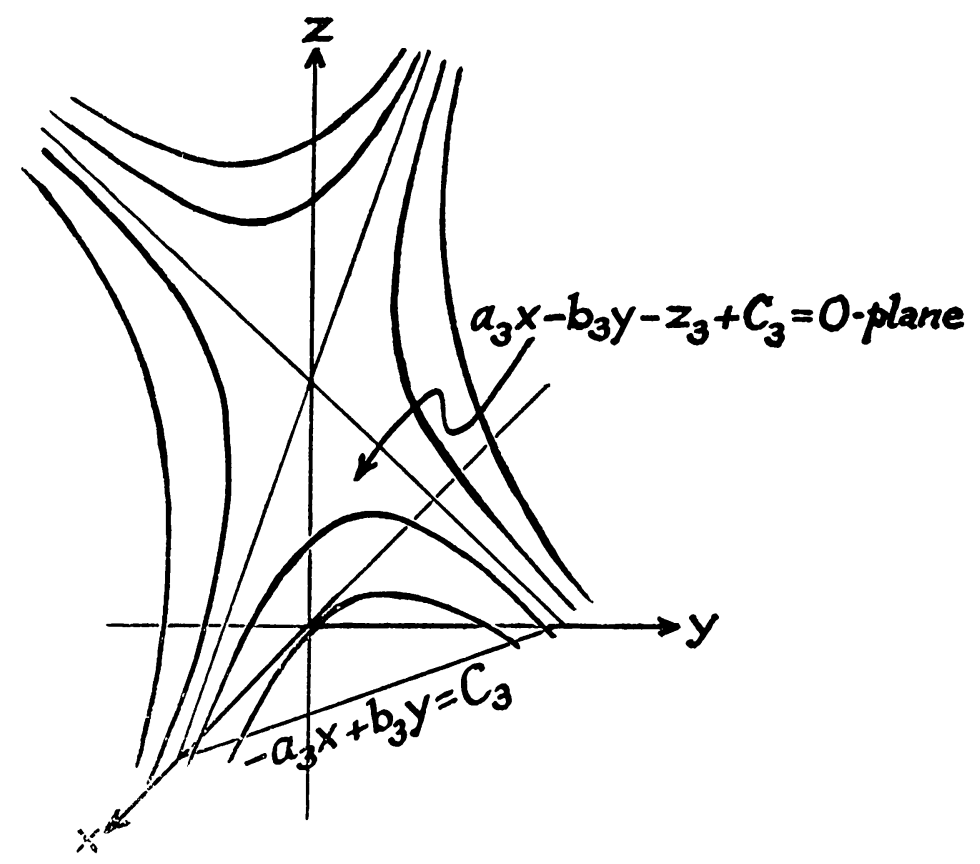

Fig. 4. The 3-dimensional orbits associated with $\mathfrak{L}_{B}$. 


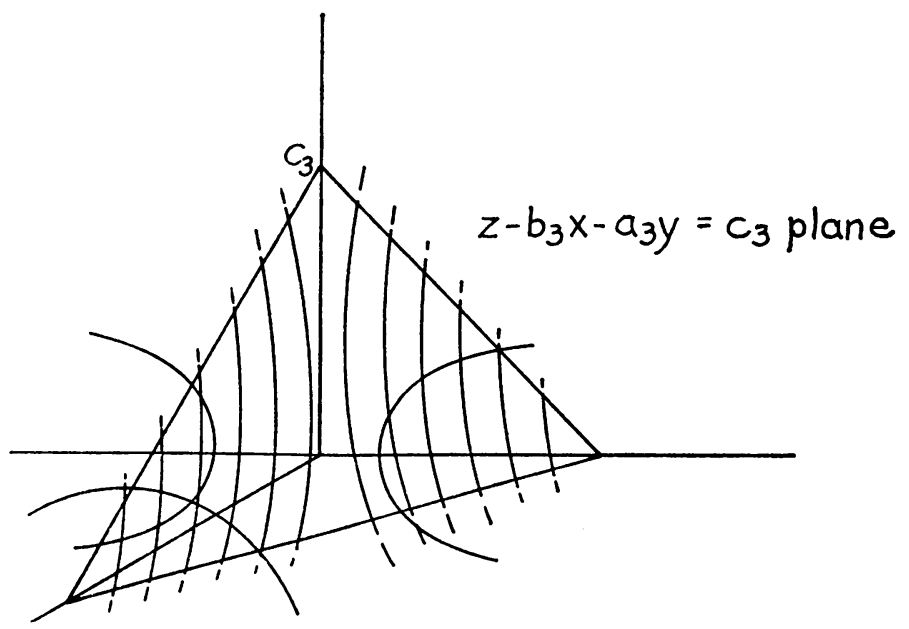

Frg. 5. Family of orbits in cortical space that are associated with $\mathfrak{L}_{b}$.

the matrices $A_{3}$ and $J_{3}$ have the respective forms

$$
A_{3}=\left(\begin{array}{lll}
1 & 0 & 0 \\
0 & 1 & 0 \\
a_{3} & b_{3} & 0
\end{array}\right), \quad J_{3}=\left(\begin{array}{lll}
1 & 0 & 0 \\
0 & 1 & 0 \\
0 & 0 & 0
\end{array}\right) .
$$

These lead in the usual way to the solution vector

$$
(x, y, z)=\left(c_{1} e^{\tau}, c_{2} e^{\tau}, a_{3} x+b_{3} y+c_{3}\right),
$$

which plots as a star of radial lines through the point $\left(0,0, c_{3}\right)$. (Fig. 6). Now $r=$ $\left(x^{2}+y^{2}\right)^{1 / 2}= \pm c\left(z-c_{3}\right) /\left(a_{3} c_{1}+b_{3} c_{2}\right)$, so that

$$
\frac{d r}{d z}=\frac{ \pm c}{a_{3} c_{1}+b_{3} c_{2}},
$$

and the flow will be upwards or downwards according as

$$
\begin{aligned}
a_{3} c_{1}+b_{3} c_{2} & >0 \\
& <0 .
\end{aligned}
$$

Thus a change in the sign of the parameters would result in a reversal of the direction of flow.

Thus the local phase portrait of $\mathscr{L}_{\mathbb{1}}$ in three-dimensional cortical space bears a marked resemblance to the morphology of the stellate type of neuron in the vicinity of the perikaryon. Since the phase portrait consists of a line of singular points, i.e., a linear arrangement of configurations like that of Fig. 6, we see that the cortical counterpart will actually be an array of stellate neurons. If now the parameters $a_{3}$ and $b_{3}$ vary, this would have the effect of changing the plane of the array. Thus the closest cortical counterpart appears to be the disk-shaped type of cortical neuron found by Colonnier [10].

We have already discussed the planar translation operators $\mathfrak{L}_{x}$ and $\mathfrak{L}_{y}$ with respect to the appearance and development of their orbits in tangential sections of the plexiform 
layer. If now we extend our discussion of these Lie derivatives to the way that they make their appearance in the depths of the cortex, the following forms for $A_{3}$ and $J_{3}$ will be required:

$$
A_{3}=\left(\begin{array}{lll}
0 & 0 & 0 \\
0 & 0 & 0 \\
a_{3} & b_{3} & 0
\end{array}\right) \quad \text { and } \quad J_{3}=\left(\begin{array}{lll}
0 & 1 & 0 \\
0 & 0 & 0 \\
0 & 0 & 0
\end{array}\right) .
$$

(Since rank $\left(A_{3}-0 \cdot I\right)^{2}=0<\operatorname{rank}\left(A_{3}-0 \cdot I\right)=1$, there is a repeated elementary divisor; hence the form of $J_{3}$.) We find in the usual way the solution vector $(u, v, w)=$ $\left(c_{1} \tau, c_{2}, c_{3}\right)$, or, in the original space,

$$
(x, y, z)=\left(c_{3}, c^{\prime}, c_{1} \tau-c_{3}\right) .
$$

This solution vector thus represents a configuration that translates vertically through cortical space as $\tau$ increases (Fig. 7). The velocity of transport is $c_{\mathbf{1}}$.

Cortical microstructures that have essentially the same appearance as the rectilinear configuration of Fig. 7 are some basal dendrites of some pyramidal neurons, lateral spines on axons, and the long fine axons that ramify horizontally for some distance in the plexiform layer. The latter give rise to an essentially rectangular grid in layers I and $\mathrm{V}$ of cat, rat, and monkey [10]; human [32], [33]; and in tangential sections of octopus brain [41]. Of course the true cortical counterparts are arrays of such neuronal processes, in the line-of-singular-points type of configuration.

Finally we consider the configuration in the cortical depths that would correspond to the Lie derivative defining the rotation group $\mathscr{L}_{r}$ and involved in the Lorentz group and the group of rotations in plane-time. The matrices $A_{3}$ and $J_{3}$ in this case take the forms

$$
A_{3}=\left(\begin{array}{rrr}
0 & 1 & 0 \\
-1 & 0 & 0 \\
a_{3} & b_{3} & 0
\end{array}\right) \quad \text { and } \quad J_{3}=\left(\begin{array}{rrr}
0 & 1 & 0 \\
-1 & 0 & 0 \\
0 & 0 & 0
\end{array}\right)
$$

The solution vector in $u, v, w$-space has the form

$$
(u, v, w)=\left(2 c_{1} \cos \left(\tau-c_{2}\right),-2 c_{1} \sin \left(\tau-c_{2}\right), 2 c_{3}\right),
$$

which, when transformed back to the original space, becomes

$(x, y, z)=\left(c_{1}\left[\cos \left(\tau-c_{2}\right)+\sin \left(\tau-c_{2}\right)\right], c_{1}\left[\cos \left(\tau-c_{2}\right)-\sin \left(\tau-c_{2}\right)\right], b_{3} x-a_{3} y+2 c_{3}\right)$.

The local phase portrait thus consists of a "line of centers" (Fig. 8). We note that the planes in which the array of families of circles lies are orthogonal to the planes of the star-shaped orbits of Fig. 6.

The cortical counterpart in this case appears to be the pericellular nests that surround the neurons of layers IV and III of the visual cortex upon which specific thalamic afferents terminate [32, Fig. 391]; [15, Fig. 71]. Such pericellular nests, having an essentially circular character, are also to be found in layer $V$ [32]. In such instances the morphology is associated with a neuron (or neurons) only transynaptically. This feature, which at first sight seems a violation of our principle that in the neighborhood of the 
cell body itself an array of neurons constitutes a cortical counterpart of a Lie group germ, in fact lends it support. A differential system such as (2) or (3) cannot, if it is to be structurally stable, have a center for a critical point. Thus if the pericellular nests were directly associated with a neuron (not merely transynaptically), this fundamental theoretical principle would be violated, and as we shall see, structural stability appears to be a vitally important requirement for a perceptual apparatus that is reproducible from creature to creature.
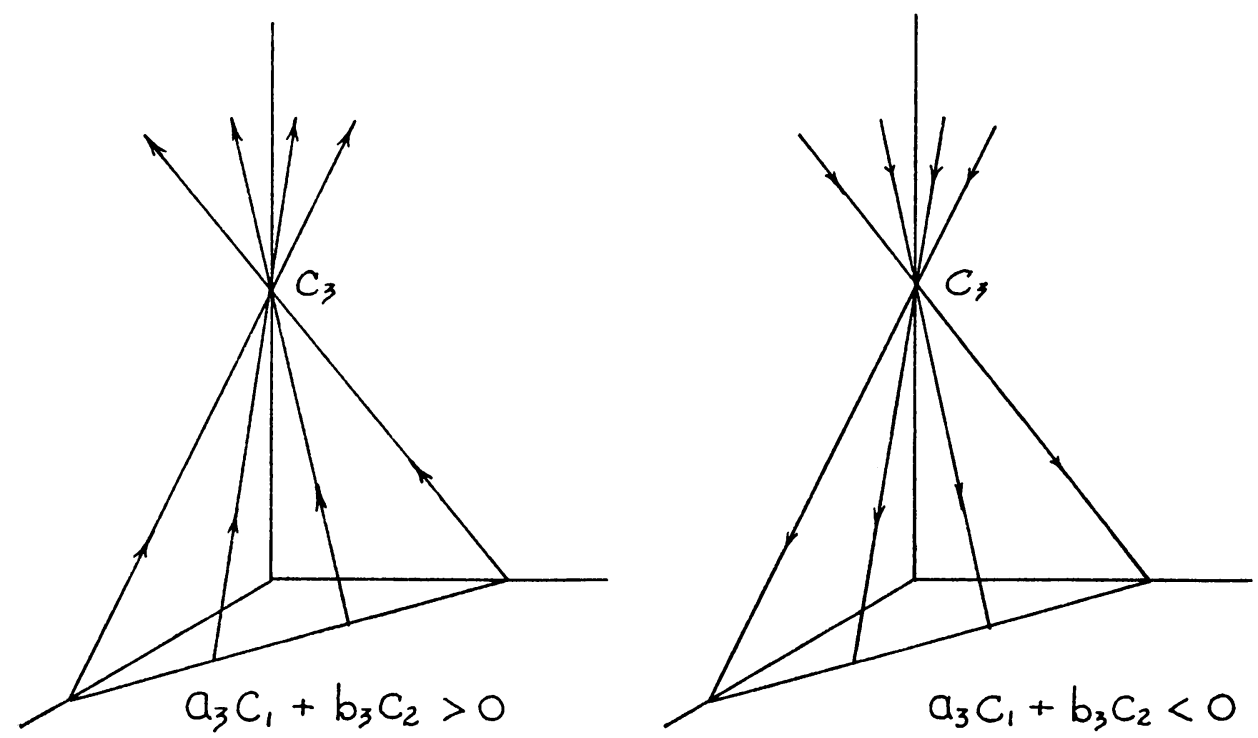

Fic. 6. Orbits in cortical space for the Lie group of size constancy defined by $\mathfrak{L}_{\text {. }}$

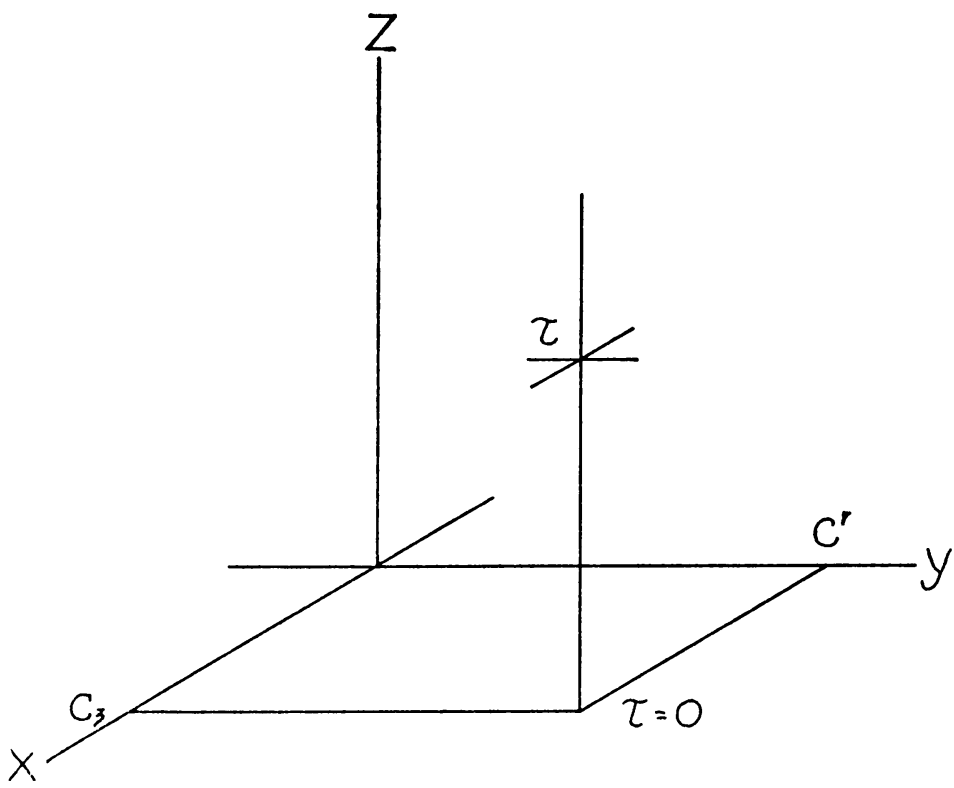

Fra. 7. Orbits in cortical space for the infinitestimal generators $\mathfrak{L}_{x}$ and $\mathfrak{L}_{y}$ of the translation groups. 


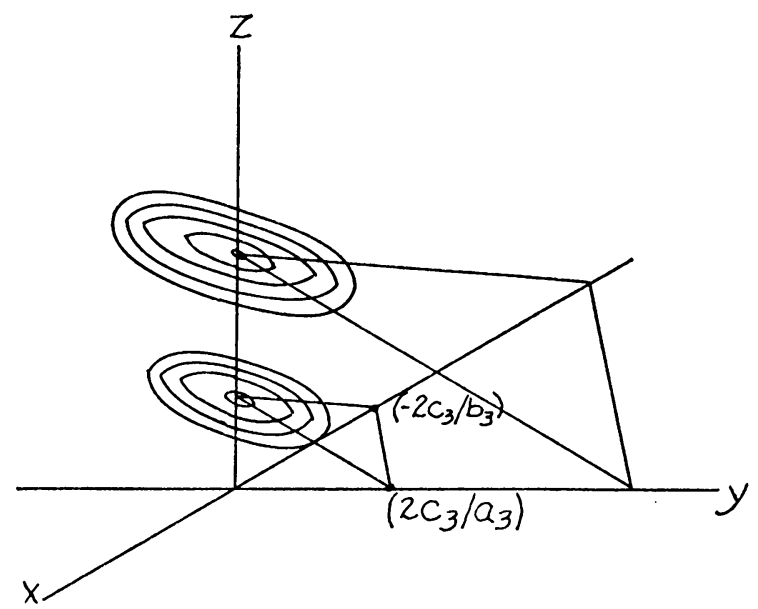

FIa. 8. Local phase portrait in cortical space of the infinitesimal transformation of the rotation group $\mathrm{O}_{2-}$

6. The role of structural stability in cortical cytoarchitecture. Let us then pass to a discussion of the question of structural stability, and how this concept, derived from the topological theory of dynamical systems, is involved in visual perception. Broadly speaking, structural stability of a system such as (2) or (3) means that its qualitative nature, i.e., its phase portrait, should not change its character under "small" topological transformations of the space and/or small perturbations of the differential system. The two-dimensional case of the structural stability problem has been analyzed at length by DeBaggis [14]. (Since the preceding configurations have been confined to arrays of planes, no loss of generality results from considering the two-dimensional situation.)

We shall first give a short resume of results for two dimensional structurally stable systems. (The effect will be to consider only one of the singular points in the "line of singular points" configuration that we encountered above.) For further results the reader is referred to Debaggis [14] and Kupka [21].

A structurally stable system can have only a finite number of singular points in any bounded domain, i.e., each singular point is isolated, and only a finite number of limit cycles as well.

The singular points of a structurally stable system can be only nodes, foci, and saddle points. A structurally stable system cannot have a center as a singular point.

Limit sets which are nodes or foci cannot, under a topological transformation, map into saddle points. The dual statement with saddle points interchanged with nodes and foci also holds.

A separatrix of a structurally stable system cannot issue from (or dually, tend toward) a saddle point and terminate in (dual: issue from) another saddle point or it itself.

The singular trajectories (sources, sinks, saddle points, and separatrices) divide the domain of a structurally stable system into a finite number of components (sets of trajectories) consisting only of ordinary trajectories.

Each component (set of trajectories) has exactly one sink and one source on its boundary.

For such structurally stable systems as possess the preceding properties, we have 
the following result. Components of such systems can only have boundaries made up of a source and a sink (Type a of Fig. 9); one source, one sink, two saddle points, and four separatrices (Type b of Fig. 9); or one source, one sink, one saddle point, and three separatrices, two of which are connected with the source (sink) and the third with the sink (source) (Type $c$ of Fig. 9). This principle should be of considerable value in interpreting interconnections among cortical neurons, although its two-dimensional nature must be taken into account in the process.

We especially note in Fig. 9 the component of Type $c$. The resemblance to Sholl's neuron types $P_{3}$ and $P_{4}$, pyramidal cells with recurrent collaterals, is very suggestive.

Nothing has yet been said about the neuropsychological significance of structural stability. If visual function is to be preserved under individual variations, that is, slight changes in anatomy from individual to individual, and a fortiori under variations from species to species, then the systems (2) or (3) must satisfy the requirement of structural stability. "Brains are as different as faces", but nonetheless we each perceive essentially the same visual image (barring such a non-topological transformation as
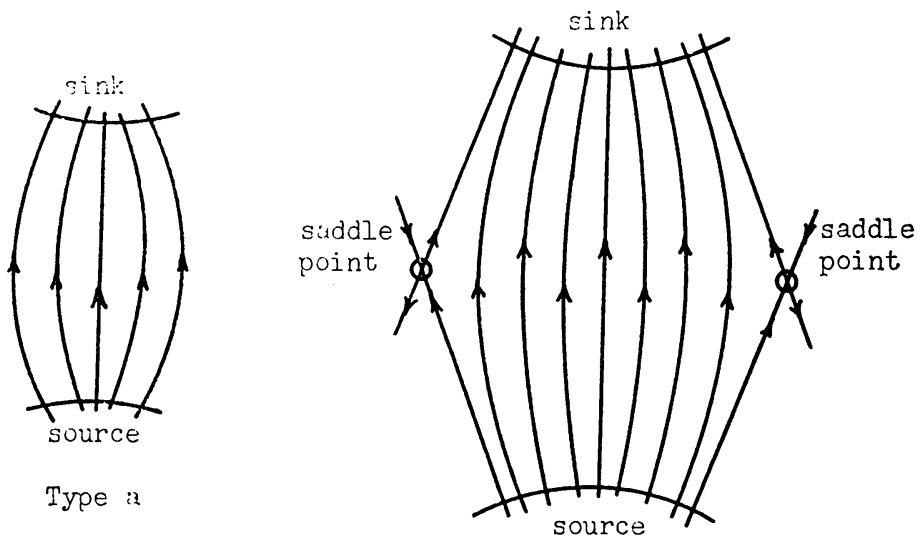

Type b

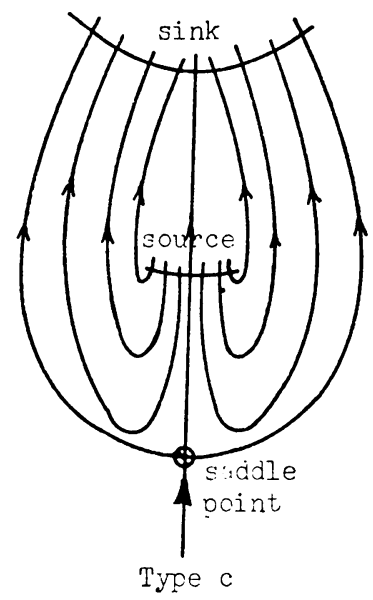

FIG. 9. Types of components (families of orbits of the same topological class) of a structurally stable system in the plane (after De Baggis [14]). 
a cortical lesion). If the system is structurally stable, then slight perturbations of its structure, especially well away from the singular point (perikaryon) will effect no significant change in its function. The latter occurs as a flow through the cortical network of neurons, and this will have the same qualitative character in the presence of perturbations and topological transformations if the system is structurally stable.

7. The Lie product, Jacobi identity, and neuron ramification. The final topic that we shall take up in connection with our discussion of neuron morphology has to do with the Lie product. Some cortical counterpart of the latter must be present if "perception by exception" is indeed a perceptual, psychological phenomenon (that is, global as well as neuronal) and if we are to have the capability of recognizing Gestalts. The connection between the local and global aspects of Lie groups has been thoroughly discussed by Palais [26], [27], and we shall say no more of this aspect of the mathematical theory here.

The Lie product is the commutator relation between two Lie derivatives

$$
\left[\mathscr{L}_{i}, \mathfrak{L}_{i}\right] f=\mathscr{L}_{i}\left(\mathfrak{L}_{i} f\right)-\mathfrak{L}_{i}\left(\mathfrak{L}_{i} f\right) .
$$

Once it has been introduced, the Lie group can be made into a Lie algebra [17]. An important property of the Lie product is that it satisfies the Jacobi identity

$$
\left[\left[\mathfrak{L}_{i}, \mathfrak{L}_{i}\right], \mathfrak{L}_{k}\right]+\left[\left[\mathfrak{L}_{k}, \mathfrak{L}_{i}\right], \mathfrak{L}_{i}\right]+\left[\left[\mathfrak{L}_{i}, \mathfrak{L}_{k}\right], \mathfrak{L}_{i}\right]=0,
$$

and all higher order identities [17, p. 114]. It is also well known that the Jacobi identity corresponds in a certain sense to an integrability condition [9], thus permitting an extension from infinitesimal transformations to Gestalten.

There appear to be many possible cortical counterparts of an antisymmetric reverberation like that of (18), notably circulating neuronal activity [39], cortical interneurons, and commissures [8]. Here, however, we shall be more concerned with the relation to neuron morphology, and the analysis will be based upon Lukasiewicz's theory of parentheses [3, p. 161]. The latter deals with the representation of a product in graphical form as follows: Consider a set of elements $A=\{a, b, c, \cdots\}$ and a product operation defined over $A$ that is nonassociative (as is the case for the Lie product-compare (19)). Then a monoid such as

$$
[(a \cdot b) \cdot c] \cdot\{[d \cdot(e \cdot f)] \cdot g\}
$$

can be represented by a "bifurcating arborescence" such as the tree in Fig. 10, wherein the number of elements emanating from any vertex is either 2 or 0 . The 0 's and 1's in parentheses below each vertex represent Lukasiewicz's scheme for taking account of a noncommutative operation (like that involved in the Lie product). This labelling orients the tree in the way indicated in the Figure, that is, from left to right and from root to top, if one associates a 0 with a pendant vertex and a 1 with all other vertices and then reads the tree in the natural order.

With this graph-theoretic tool at hand, we shall investigate how it would apply to the Jacobi identity (and the extensions thereof), and then relate the result to neuron morphology. To this end we write the Jacobi identity in abstract form, as follows:

$$
[[\alpha, \beta], \gamma]+[[\gamma, \alpha], \beta]+[[\beta, \gamma], \alpha]=0 \text {. }
$$

Now each of the Lie products involved in this sum has a vector character [17, Theorem 6-7 and Eq. (6-23)]. The vector sum in (20) adds to zero, and so must represent a closed 


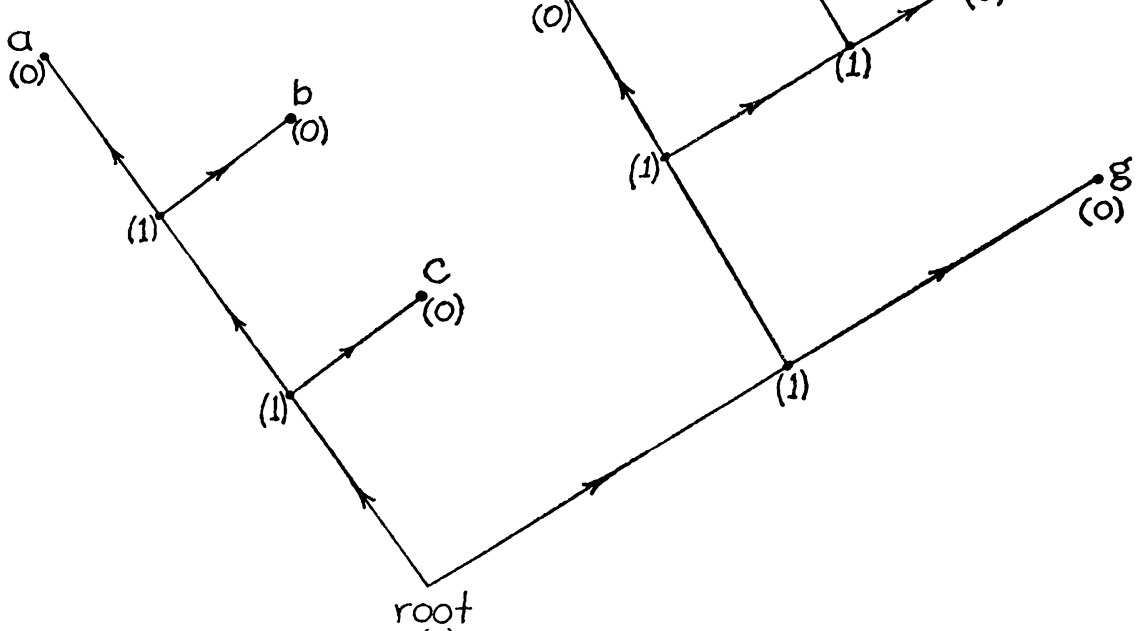

(1)

FIg. 10. A tree representing $[(a \cdot b) \cdot c] \cdot\{[d \cdot(e \cdot f)] \cdot g\}$, according to Lukasiewicz's theory of parentheses (after Berge [3]).

triangular configuration. Each vertex of the triangle will have associated with it a monoid, like that represented in Fig. 10, of the form $\left[\left[\alpha_{1}, \alpha_{2}\right], \alpha_{3}\right]$. Thus we may represent the Jacobi identity (20) graphically in the form shown in Fig. 11, where the orientation is taken as positive clockwise and out-to-in. It is interesting to note that the neurofibrils within a neuron apparently exhibit this same morphology.

The Jacobi identity of next higher order [17, p. 114] has the form

$$
[[[\alpha, \beta], \gamma], \delta]+[[[\beta, \alpha], \delta], \gamma]+[[[\gamma, \delta], \alpha], \beta]+[[[\delta, \gamma], \beta], \alpha]=0 .
$$

We see that one additional vector occurs in the closed configuration. It can appear between any successive pair of vertices of the closed triangle in the center of Fig. 11. One possible arrangement that is at the same time consistent with its precursor in Fig. 11 is shown in Fig. 12. Numerous other arrangements can also be obtained through use of the anticommutative property of the Lie product

$$
[\alpha, \beta]=-[\beta, \alpha]
$$

and the other form of the Jacobi identity

$$
[\alpha,[\beta, \gamma]]+[\gamma,[\alpha, \beta]]+[\beta,[\gamma, \alpha]]=0 .
$$

We thus see that as the Jacobi identity is extended to include more and more Lie derivatives, additional "dendrites" are formed and additional "rami" occur, thus agreeing with not only neuron morphology but also with the course of development of the latter in the neonatal cortex of kitten [31] and human infant [11]. The more complex the dendritic branching and extension, the higher the order of the integrability condition expressed thereby. This feature, too, parallels the development of the ability to perceive larger and more complex Gestalten during the course of infancy. 


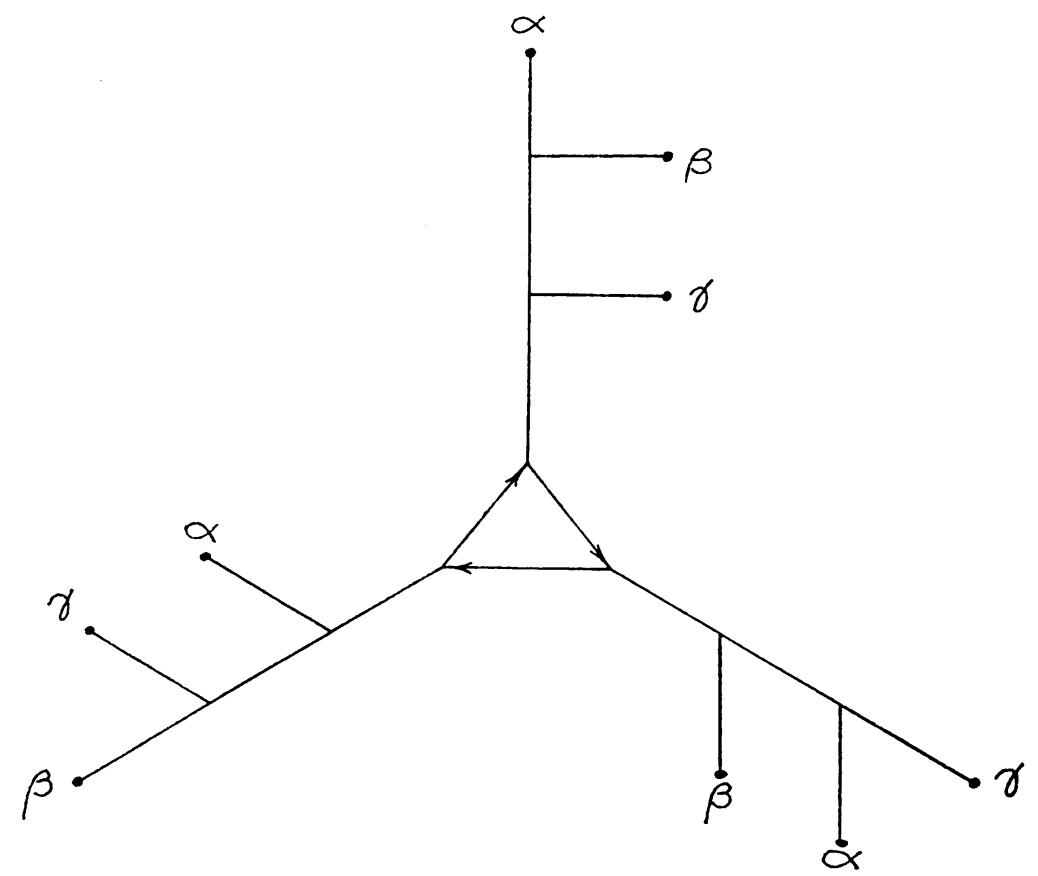

FIG. 11. Representation of the Jacobi identity as a tree.

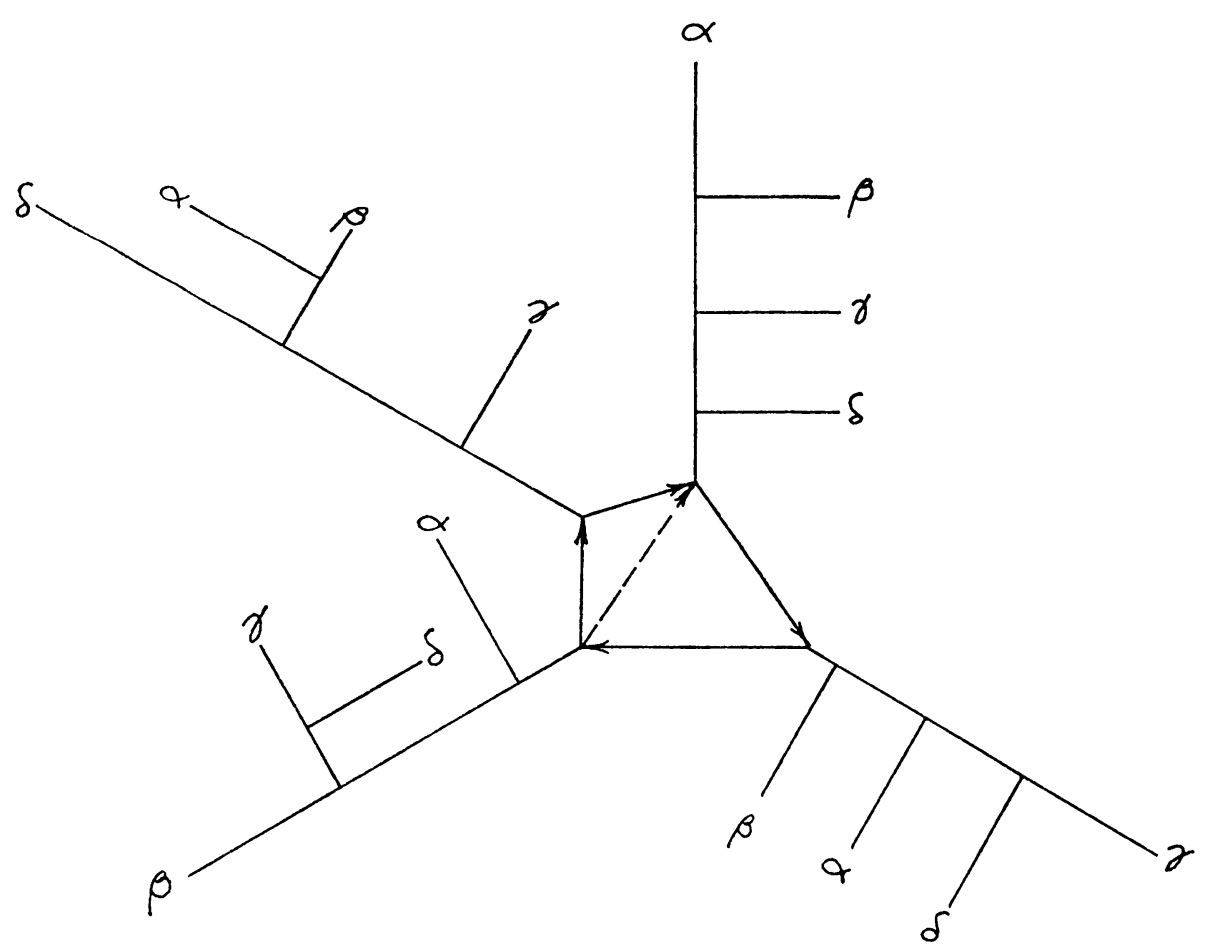

Fia. 12. A tree representing the 4th order Jacobi identity derived from Lukasiewicz's theory of parentheses. 


\section{REFERENCES}

1. Y. Akishige, Theoretical considerations on the problems of perceptual constancies, Kyushu Psychol. Studies No. II, 1-57 (1961)

2. Y. Akishige, Perceptual constancy and the law of conservation of perceptual information, Kyushu Psychol. Studies No. III, 1-48 (1965)

3. C. Berge, The Theory of Graphs and Its Applications, Wiley and Sons, Inc., New York, 1962

4. G. H. Bishop, and J. L. O'Leary. Potential records from the optic cortex of the cat, J. Neurophysiol. 1, 391-404 (1938)

5. G. H. Bishop, Natural history of the nerve impulse, Physiol. Rev. 36, 376-399 (1956)

6. T. H. Bullock, Neuron doctrine and electrophysiology, Science 129, 997-1002 (1959)

7. E. Cassirer, The concept of group and the theory of perception, Philos. and Phenomenological Res. 5, 1-36 (1944)

8. H. T. Chang, Changes in excitability of cerebral cortex following single electrical shock applied to cortical surface, J. Neurophysiol. 14, 95-111 (1951)

9. P. M. Cohn, Lie groups, Cambridge Univ. Press, 1957

10. M. Colonnier, The tangential organization of the visual cortex, J. Anatomy (London) 98, 327-344 (1964)

11. J. L. Conel, The postnatal development of the human cerebral cortex. I; Cortex of the newborn. II; Cortex of the one-month infant. III; Cortex of the three-month infant. IV; Cortex of the six-month infant. V; Cortex of the fifteen-month infant. VI; Cortex of the 24-month infant. VII; Cortex of the four year old child, Harvard University Press, 1939, 1941, 1941, 1955, 1955, 1959, 1959

12. J. T. Culbertson, A neural analysis of behavior and consciousness, Wm. C. Brown Co., Dubuque, Iowa, 1950

13. J. T. Culbertson, Some uneconomical robots, in Automata Studies, C. E. Shannon and J. T. McCarthy, Eds., Princeton Univ. Press, Princeton, N. J., 1956, pp. 99-116

14. H. F. DeBaggis, Dynamical systems with stable structures, in Contributions to the Theory of Nonlinear Oscillations, Vol. II, S. Lefschetz, Ed., Princeton Univ. Press, Princeton, N. J., 1952, pp. 37-59

15. J. F. Fulton, Physiology of the nervous system, 3rd ed., Oxford Univ. Press, New York, 1949

16. M. Gihr, Methode zur Rekonstruktion von Nervenzellen, J. f. Hirnforschung 5, 7-22 (1962)

17. H. W. Guggenheimer, Differential geometry, McGraw-Hill, New York, 1963

18. W. C. Hoffman, The Lie algebra of visual perception, J. Math. Psychol. 3, 65-98 (1966)

19. W. C. Hoffman, Contments on 'optical art', Applied Optics 5, 873-874 (1966)

20. M. Kucharzewski, and M. Kuczma. Basic concepts of the theory of geometric objects, Rozprawy Matematyczne XLIII, Warsaw, 1964

21. I. Kupka, The singularities of integrable structurally stable Pfaffian forms, Proc. Nat. Acad. Sci. U.S.A. 52, 1431-1432 (1964)

22. C-L. Li, C. Cullen, and H. H. Jasper, Laminar microelectrode studies of specific somatosensory cortical potentials, J. Neurophysiol. 19, 111-130 (1956)

23. P. Mendelson, On phase portraits of critical points in $n$-space, Contributions to the Theory of Nonlinear Oscillations, Vol. IV, S. Lefschetz, Ed., Princeton Univ. Press, Princeton, N. J., 1958, pp. 167-199

24. K. Motokawa, Mechanisms for the transfer of information along the visual pathways, in Internat. Rev. Neurobiol. 5, C. C. Pfeiffer and J. R. Smythies, Eds., Academic Press, N. Y., 1963, pp. 121-181.

25. J. R. Munkres, Elementary differential topology, Princeton Univ. Press, Princeton, N. J., 1963

26. R. S. Palais, Global formulation of the Lie theory of transformation groups, Mem. Amer. Math. Soc., No. 22, 1957

27. R. S. Palais, The classification of $G$-spaces, Mem. Amer. Math. Soc., No. 36, 1960

28. W. Pitts, and W. S. McCulloch. How we know universals. The perception of auditory and visual forms, Bull. Math. Biophys. 9, 127-147 (1947)

29. K. H. Pribram, A review of theory in physiological psychology, in Annual Revs. Psychol., Vol. 11, Palo Alto, Calif., 1960, pp. 1-40

30. K. H. Pribram, The new neurology: memory, novelty, thought and choice, in EEG and behavior, G. H. Glaser, Ed., Basic Books, N. Y., 1963, pp. 149-173

31. D. P. Purpura, Morphological basis of elementary evoked response patterns in the neocortex of the newborn cat, Annals N. Y. Acad. Sci., Vol. 92, Art. 3 (1961), pp. 840-859

32. S. Ramon y Cajal, Histologie du systeme nerveux de l'homme et des vertebres, Vol. II, Madrid, 1955 
33. S. Ramon y Cajal, Studies on vertebrate neurogenesis, Thomas, Springfield, Ill., 1960

34. N. Rashevsky, Mathematical biophysics: physico-mathematical foundations of biology, Vol. II, 3rd rev. ed., Dover, N. Y., Chapter 11

35. L. E. Reizin', Behavior of the integral curves of a system of differential equations near a singular point in a space of more than one dimension, Amer. Math. Soc. Transl. (2) Vol. 18, Amer. Math. Soc., Providence, R. I., 1961, pp. 173-186

36. F. Rosenblatt, Perceptual generalization over transformation groups, in Self-Organizing Systems, M. Yovits and S. Cameron, Eds., Pergamon Press, 1960

37. D. A. Sholl, The organization of the cerebral cortex, Wiley \& Sons, Inc., New York, 1956

38. J. R. Smythies, The stroboscopic patterns. II. The phenomenology of the bright phase and after-images, British J. Psychol. 50, 305-324 (1959)

39. M. Verzeano, and K. Negishi, Neuronal activity in cortical and thalamic networks, J. General Physiol. 43, Suppl., 177-195 (1960)

40. N. Wiener, Cybernetics, 2nd ed., MIT Press, Camb., Mass., 1961, Ch. VI: gestalt and universals

41. J. Z. Young, Two memory stores in one brain, Endeavour 24, 13-20 (1965) 